\title{
Initial Value Semiclassical Approaches to Reactive and Non Reactive Transition Probabilities
}

\author{
N. Faginas Lago and A. Laganà \\ Dipartimento di Chimica, Università di Perugia, Via Elce di Sotto, 8, \\ 06123 Perugia, Italy
}

\begin{abstract}
Semiclassical initial value representations (IVR) of the S matrix elements for state to state transitions provide a practical way for incorporating quantum mechanical effects into the classical estimate of the transition probability of elementary chemical processes. This semiclassical IVR approach applies to the study of the reactive and non-reactive transitions in atom diatom collisions. As a case study the collinear $\mathrm{H}+$ $\mathrm{Cl}_{2}$ reaction is considered.
\end{abstract}

\section{Introduction}

Molecular dynamics calculations are becoming a kind of universal tool to rationalize the behaviour of chemical processes. However, most often, they are performed using classical mechanics that can not reproduce quantum effects. Accordingly, highly inaccurate results are obtained when interference, tunneling and resonance effects are important. On the other hand, quantum calculations are exact but difficult to carry out especially when dealing with large systems. As an alternative to quantum techniques semiclassical approaches are often used. Semiclassical approaches retain the simplicity of classical mechanics (they are based on the integration of classical trajectories) while introducing quantum-like corrections by exploiting the information carried by the classical phase.

A key problem of the traditional semiclassical formulations is that they require the solution of a two point boundary problem and the numerical search for related root trajectories. This makes the search for semiclassical contributions to the transition probabilty cumbersome and unsuitable for parallel and distributed computing. In this paper has been adopted the more recent initial value representation (IVR) approach [1] that avoids the search for root trajectories by properly mapping the formulation of the $\mathrm{S}$ matrix elements into the space of the initial variables.

In order to more easily tackle the problem of dealing with reactive transitions on which the literature (see ref. [1], and references therein) is rather poor our investigation was focused on atom diatom collinear systems and on the possibility of using a unified approach for both reactive and non reactive transitions. The paper is articulated as follows: in Section 2 the IVR semiclassical method is outlined; in Section 3 the specialization of the method for collinear systems is given; in Section 4 the $\mathrm{H}+\mathrm{Cl}_{2}$ reactive case study is illustrated; in Section 
5 a comparison of exact quantum and IVR semiclassical product vibrational distributions is carried out.

\section{The IVR Semiclassical S Matrix}

The basic semiclassical approach [1] pivots on the definition of the matrix element of the time evolution operator (propagator) $K_{1 \rightarrow 2}(t)$ that for the atom diatom case reads

$$
\begin{aligned}
K_{1 \rightarrow 2}(t) \equiv & \left\langle\psi_{2}\left|e^{-i H t / \hbar}\right| \psi_{1}\right\rangle= \\
& \int d \mathbf{x}_{1} \int d \mathbf{x}_{2} \psi_{2}^{*}\left(\mathbf{x}_{2}\right) \psi_{1}\left(\mathbf{x}_{1}\right)\left\langle\mathbf{x}_{2}\left|e^{-i H t / \hbar}\right| \mathbf{x}_{1}\right\rangle
\end{aligned}
$$

which is the probability amplitude for a transition from the bound initial state 1 (described by the wavefunction $\psi_{1}$ in the coordinate $\mathbf{x}_{1}$ at time zero) to the bound state 2 (described by the wavefunction $\psi_{2}$ in the coordinate $\mathbf{x}_{2}$ at time $t$ ) for a system whose Hamiltonian is $H$. Using the standard semiclassical approximation for the coordinate representation of the propagator one has

$$
\begin{aligned}
K_{1 \rightarrow 2}(t) & =\sum_{\text {roots }} \int d \mathbf{x}_{1} \int d \mathbf{x}_{2} \psi_{2}\left(\mathbf{x}_{2}\right)^{*} \psi_{1}\left(\mathbf{x}_{1}\right) \\
& \times\left[(2 \pi i \hbar)^{F}\left|\frac{\partial \mathbf{x}_{2}}{\partial \mathbf{P}_{1}}\right|\right]^{-1 / 2} e^{i S_{t}\left(\mathbf{x}_{2}, \mathbf{x}_{1}\right) / \hbar}
\end{aligned}
$$

where $F$ is the number of degrees of freedom and, $P_{i}$ is the momentum of the $i$ th state. $S_{t}\left(\mathbf{x}_{2}, \mathbf{x}_{1}\right)$ is the classical action associated with the trajectory connecting $\mathbf{x}_{1}$ to $\mathbf{x}_{2}$ in time $t$ that can be formulated as

$$
S_{t}\left(\mathbf{x}_{2}, \mathbf{x}_{1}\right)=\int_{0}^{t} d t^{\prime} \mathbf{P}\left(t^{\prime}\right) \dot{\mathbf{x}}\left(t^{\prime}\right)-H\left(\mathbf{P}\left(t^{\prime}\right), \mathbf{x}\left(t^{\prime}\right)\right)
$$

where primed quantities are related to products and dotted quantities are time derivatives.

The Jacobian factor in Eq. (2)

$$
\left|\frac{\partial \mathbf{x}_{2}}{\partial \mathbf{P}_{1}}\right|=\left|\frac{\partial \mathbf{x}_{t}\left(\mathbf{P}_{1}, \mathbf{x}_{1}\right)}{\partial \mathbf{P}_{1}}\right|
$$

is evaluated at the roots, that is at the solutions of the nonlinear two boundary value problem

$$
\mathbf{x}_{t}\left(\mathbf{P}_{1}, \mathbf{x}_{1}\right)=\mathbf{x}_{2}
$$

As already mentioned, in general, there are multiple roots since $\mathbf{x}_{t}$ needs not to be a monotonic function of $\mathbf{P}_{1}$ and the summation in Eq. (2) is over all such roots. This process is often unstable (especially when both reactive and non reactive events can take place) and disrupts concurrency in trajectory calculations. 
To eliminate the need for carrying out the root finding process, the IVR approach maps the final position independent variable $\mathbf{x}_{2}$ into the initial variable $\mathbf{P}_{1}$ and evaluates numerically the integrals of Eq. (2). In addition, the propagator in the Cartesian coordinates or momentum representation [3] is substituted by its semiclassical formulation. This leads to the expression

$$
\begin{aligned}
K_{1 \rightarrow 2}(t)= & \int d \mathbf{x}_{1} \int d \mathbf{P}_{1}\left[\left|\frac{\partial \mathbf{x}_{t}\left(\mathbf{x}_{1}, \mathbf{P}_{1}\right)}{\partial \mathbf{P}_{1}}\right| /\right. \\
& \left.(2 \pi i \hbar)^{F}\right]^{1 / 2} e^{i S_{t}\left(\mathbf{x}_{1}, \mathbf{P}_{1}\right) / \hbar} \psi_{2}\left(\mathbf{x}_{t}\right)^{*} \psi_{1}\left(\mathbf{x}_{1}\right)
\end{aligned}
$$

in which $S_{t}\left(\mathbf{x}_{1}, \mathbf{P}_{1}\right) \equiv S_{t}\left(\mathbf{x}_{t}\left(\mathbf{x}_{1}, \mathbf{P}_{1}\right), \mathbf{x}_{1}\right), \mathbf{x}_{2}\left(\mathbf{x}_{1}, \mathbf{P}_{1}\right)$ is written in a more general form as $\mathbf{x}_{t}\left(\mathbf{x}_{1}, \mathbf{P}_{1}\right)$, the double ended boundary condition of Eq. (4) has been replaced by an integral over the phase space of the initial conditions and the Jacobian factor $\left(\partial \mathbf{x}_{t} / \partial \mathbf{P}_{1}\right)$ appears in the numerator (rather than in the denominator as is in Eq. (2)) avoiding so far possible singularities.

\section{The Collinear Atom Diatom Case Study}

To carry out the numerical evaluation of the semiclassical $\mathbf{S}$ matrix we have simplified the problem by considering the atom diatom collinear processes of the type $\mathrm{A}+\mathrm{BC}\left(\nu_{i}\right) \rightarrow \mathrm{AB}\left(\nu_{f}\right)+\mathrm{C}$ (reactive) and $\mathrm{A}+\mathrm{BC}\left(\nu_{i}\right) \rightarrow \mathrm{A}+\mathrm{BC}\left(\nu_{f}\right)$ (non reactive). The classical collinear atom diatom Hamiltonian function reads as

$$
H=\frac{1}{2 \mu_{A, B C}} P_{R}^{2}+\frac{1}{2 \mu_{B C}} P_{r}^{2}+V(R, r)
$$

where $V(R, r)$ is the potential of the system $R$ and $r$ the reactant Jacobi coordinates, $P_{R}$ and $P_{r}$ are the related momenta, $\mu_{B C}$ and $\mu_{A, B C}$ the reduced masses of the diatom and of the atom-diatom system. Classical trajectories were computed by integrating the following set of Hamilton equations

$$
\begin{aligned}
& \dot{R}=P_{R} / \mu_{A, B C} \\
& \dot{r}=P_{r} / \mu_{B C} \\
& \dot{P_{R}}=-\partial V / \partial R \\
& \dot{P}_{r}=-\partial V / \partial r
\end{aligned}
$$

(where $\dot{R}, \dot{P}_{R}, \dot{r}$ and $\dot{P}_{r}$ are the time derivatives of $R, P_{R}, r$ and $P_{r}$, respectively) using a fourth order Runge-Kutta method and adjusting the stepsize to guarantee energy conservation and collision time constance. 
To compute the phase associated with each trajectory, the following fifth differential equation was integrated

$$
\dot{S}_{t}=-\omega \dot{n}-R \dot{P}_{R}
$$

where $S_{t}$ is the already mentioned classical action formulated in terms of the action angle variables $J$ and $\omega$ (defined as $\omega=\int \partial P_{r}(J) / \partial J d r$ and $2 n\left(n+\frac{1}{2}\right) \hbar=$ $J=\oint P_{r} d r[4]$ with $n$ being the classical vibrational number continuously varying through the integer values of its discrete quantum analogue $\nu$ and $\omega$ being the oscillator phase angle).

The collision is started at a value of $R$ sufficiently large $\left(R_{0}\right)$ that makes the atom diatom interaction negligible. The quantum number $\nu_{i}$ for the transition $1 \rightarrow 2$ determines the initial value of the classical number $n_{i}$ and hence the initial value of $P_{R}\left(P_{R_{0}}\right)$ by energy conservation. The initial value of $\omega\left(\omega_{0}\right)$, to which corresponds uniquely a value of $r\left(r_{0}\right)$, is chosen on a regular grid in the range $[-0.5-0.5]$. Thus the initial boundary conditions are

$$
\begin{aligned}
& R_{0}=\text { Large } \\
& P_{R_{0}}=-\left\{2 \mu_{A, B C}\left[E-\varepsilon_{n_{i}}\right]\right\}^{1 / 2} \\
& r_{0}=r\left(\omega_{0}, n_{i}\right) \\
& P_{r_{0}}=P_{r}\left(\omega_{0}, n_{i}\right)
\end{aligned}
$$

where $E$ is the total energy of the system and $\varepsilon_{n_{i}}$ the initial vibrational energy of the diatom in the state $n_{i}=\nu_{i}$.

In the procedure illustrated above, the use of action angle variables is motivated by the need of carrying out a proper selection of initial conditions that is more satisfactory if the oscillator phase is sampled uniformly. For this reason, our computational procedure first selects the initial values of the action angle variables of state 1 on a regular grid, then carries out the integration of the Hamilton equations using the Jacobi coordinates and finally transforms back all quantities into the action angle variables of state 2 .

For our calculations we have considered the diatoms as a Morse oscillators, since this makes it possible to work out analytically the relationships linking the action angle variables to the other variables.

The IVR formulation of the semiclassical $\mathbf{S}$ matrix can then be obtained by expressing it in terms of the propagator and integrating over time $t$. The IVR $\mathrm{S}$ matrix element $\left(S_{1 \rightarrow 2}^{I V R}(E)\right)$ then reads [5]

$$
\begin{aligned}
S_{1 \rightarrow 2}^{I V R}(E)= & -e^{-i\left(k_{1} R_{1}+k_{2} R_{2}\right)} \int d P_{r_{0}} \int d r_{0} \int d P_{R_{0}} \\
& \times\left[\left|\frac{\partial\left(r_{t}, R_{t}\right)}{\partial\left(P_{r_{0}}, P_{R_{0}}\right)}\right| /(2 \pi i \hbar)^{F}\right]^{1 / 2} e^{i\left[E t+S_{t}\left(P_{r_{0}}, r_{0}, P_{R_{0}}, R_{0}\right)\right] / \hbar} \\
& \psi_{2}\left(r_{t}\right) \psi_{1}\left(r_{0}\right) \hbar \sqrt{k_{1} k_{2}} / P_{R_{t}}
\end{aligned}
$$


with $\mu$ being the reduced mass of the system and $R_{t}$ and $r_{t}$ the value of the Jacobi coordinates obtained at time $t$ by integrating Eqs. 12 starting from the initial conditions $R_{0}, r_{0}, P_{R_{0}}$ and $P_{r_{0}}$. The two $k_{j}(j=1,2)$ factors are the initial and final wave numbers defined as

$$
k_{j}=\sqrt{2 \mu\left(E-\varepsilon_{j}\right) / \hbar^{2}} .
$$

Finally, the detailed probability $P_{1 \rightarrow 2}(E)$ of the 1 to 2 transition is worked out as usual from the related $S_{1 \rightarrow 2}^{I V R}(E)$ element by taking its square modulus

$$
P_{1 \rightarrow 2}(E)=\left|S_{1 \rightarrow 2}^{I V R}(E)\right|^{2} .
$$

\section{The $\mathrm{H}+\mathrm{Cl}_{2}$ Case Study}

The potential $V$ adopted for the $\mathrm{H}+\mathrm{Cl}_{2}$ system is of the LEPS type

$$
V\left(r_{A B}, r_{B C}, r_{A C}\right)=\sum_{i=1}^{3} Q_{i}-\left\{\sum_{i \leq j}^{3}\left(J_{i}-J_{j}\right)^{2} / 2\right\}^{1 / 2}
$$

with

$$
\begin{gathered}
Q_{i}=\left\{{ }^{1} E_{i}-\frac{1-\Delta_{i}}{1+\Delta_{i}} E_{i}\right\} / 2 \\
J_{i}=\left\{{ }^{1} E_{i}+\frac{1-\Delta_{i}}{1+\Delta_{i}} E_{i}\right\} / 2 \\
{ }^{1} E_{i}=D_{i} \exp \left[-\beta_{i}\left(r_{i}-r_{i}^{e}\right)\right]\left\{\exp \left[-\beta_{i}\left(r_{i}-r_{i}^{e}\right)\right]-2\right\} \\
{ }^{3} E_{i}=\frac{1}{2} D_{i} \exp \left[-\beta_{i}\left(r_{i}-r_{i}^{e}\right)\right]\left\{\exp \left[-\beta_{i}\left(r_{i}-r_{i}^{e}\right)\right]+2\right\}
\end{gathered}
$$

where the index $i$ extends over all diatomic pairs (AB, BC and AC) and $r_{i}$ is the interatomic distance of the $i$ th diatom. The value of the parameters $D_{i}, r_{i}^{e}, \beta_{i}$ and $\Delta_{i}$ (for the $\mathrm{H}+\mathrm{Cl}_{2}$ system [6]) are given in Table 1.

Table 1. Value of the LEPS parameters for $\mathrm{HCl}$ and $\mathrm{Cl}_{2}$

\begin{tabular}{c|c|c|c|c|} 
& $\beta_{i}\left(\AA^{-1}\right)$ & $r_{i}^{e}(\AA)$ & $\Delta_{i}$ & $\mathrm{D}_{i}(\mathrm{eV})$ \\
\hline $\mathrm{HCl}$ & 1.86932 & 1.273200 & 0.067000 & 4.625970 \\
$\mathrm{Cl}_{2}$ & 2.008020 & 1.999800 & -0.113000 & 2.516970
\end{tabular}


Among practical advantages of using the LEPS potential is the fact that the asymptotic diatom quantum eigenenergies have the closed form expression

$$
\varepsilon_{\nu}=-D\left[1-\frac{\hbar \beta}{(2 \mu D)^{1 / 2}}\left(\nu+\frac{1}{2}\right)\right]^{2}
$$

where, as usual, $\nu$ is the vibrational quantum number and the angle variable $\omega$ is related to the internuclear distance $r$ by the relationship

$$
r-r^{e}=\frac{1}{\beta} \ln \left[\frac{1-\left(1+\varepsilon_{\nu} / D\right)^{1 / 2} \cos (2 \pi \omega)}{\left(-\varepsilon_{\nu} / D\right)}\right] .
$$

The vibrational wave function $\phi_{\nu}(r)$ has the following form

$$
\phi_{\nu}(r)=N_{\nu} e^{-z / 2} z^{c / 2} L_{\nu}^{c}(z)
$$

where

$$
\begin{gathered}
N_{\nu}=[\beta c \nu ! / \Gamma(k-\nu)]^{1 / 2}, \\
c=k-2 \nu-1, \\
k=2\left(2 \mu_{B C} D\right)^{1 / 2} / \beta \hbar \\
z=k e^{-\beta\left(r-r^{e}\right)}
\end{gathered}
$$

with $L_{\nu}^{c}(z)$ being the Laguerre polynomial and $\Gamma(i)$ the Gamma function [7].

\section{Product Vibrational Distributions}

As already mentioned, non reactive processes are the most consolidated case study for semiclassical approaches and the most relevant for Molecular Dynamics calculations. The peculiarity of the case considered here is that the LEPS potential includes the reactive channel and, therefore, it highly distorts the triatom even when reaction does not occur and when reactive processes coexist with non reactive ones. This makes of particular interest the study of the near the threshold behaviour. A preliminary study of the results obtained for the $\mathrm{H}+$ $\mathrm{Cl}_{2}$ system is given in [8] where an analysis of the convergence with the number of integrated trajectories is performed. Here, an extended analysis of the accuracy of state to state IVR semiclassical probabilities for both reactive and non reactive processes is given.

The accuracy of semiclassical results is tested against quantum results by comparing the structure of the calculated product vibrational distribution (PVD). For illustrative purposes we report here also quasiclassical values determined by assigning the classical result to the closest quantum state and then taking the ratio between the number of trajectories assigned to the considered quantum final state and the total number of integrated trajectories. 
In Fig. 1 the non reactive PVDs calculated for values of the collision energy $\left(E_{t r}\right)$ extending up to $1.26 \mathrm{kcal} / \mathrm{mol}$ (the threshold energy) are given. As apparent from the figure quasiclassical probabilities are always 1 for the elastic transitions and 0 for the inelastic ones. On the contrary, semiclassical IVR values (empty squares connected by dashed dotted lines) reproduce much better the structure of quantum (solid diamonds connected by dashed lines) probabilities. The deviation of semiclassical IVR from quantum results of the PVD does not usually exceed $10 \%$ although there is no clear systematic for the related error.

At energies above the threshold the product channel becomes accessible and the probability of populating some product vibrational states differs from zero. Reactive state to state semiclassical IVR probabilities calculated at $\nu_{i}=0$ (upper row) and $\nu_{i}=1$ (lower row) are plotted (dashed dotted line) in Fig. 2 as product vibrational distributions (as a function of $\left(\left(\nu_{f}\right)\right.$ at increasing value of the total energy (from the left hand side to the right hand side). For comparison also exact quantum (dashed line) and quasiclassical ones (solid line) are shown in the same figure.

The figure shows that differences between reactive probabilities calculated using different methods can be quite large. In particular, the deviation of quasiclassical results is significant when product vibrational distributions show a definite structure (like the bimodal shape obtained at $\nu_{i}=1$ ). On the contrary, the agreement between semiclassical IVR and quantum PVDs is definitely better in particular because they reproduced the related structure is when there is more than one maximum.

\section{Conclusions}

The significant progress made by Molecular Dynamics is prompting the introduction of more accuracy in calculating state to state transition probabilities when classical mechanics dynamical techniques are used. In this paper numerical tests of the traditional way of building quantum corrections into classical mechanics approaches through the calculation of the classical phase associated with the trajectories described by the system and the evaluation from it of semiclassical S matrix elements have been discussed. In particular, the recently proposed IVR formulation of the semiclassical $\mathrm{S}$ matrix has been considered because of its straightfarword concurrent implementation. Results of the test are extremely encouraging and plans are being made for its implementation on metacomputer and grid platforms.

\section{Acknowledgments.}

This research has been financially supported by MIUR, ASI and CNR (Italy) and COST in Chemistry Action D23 (Europe). 

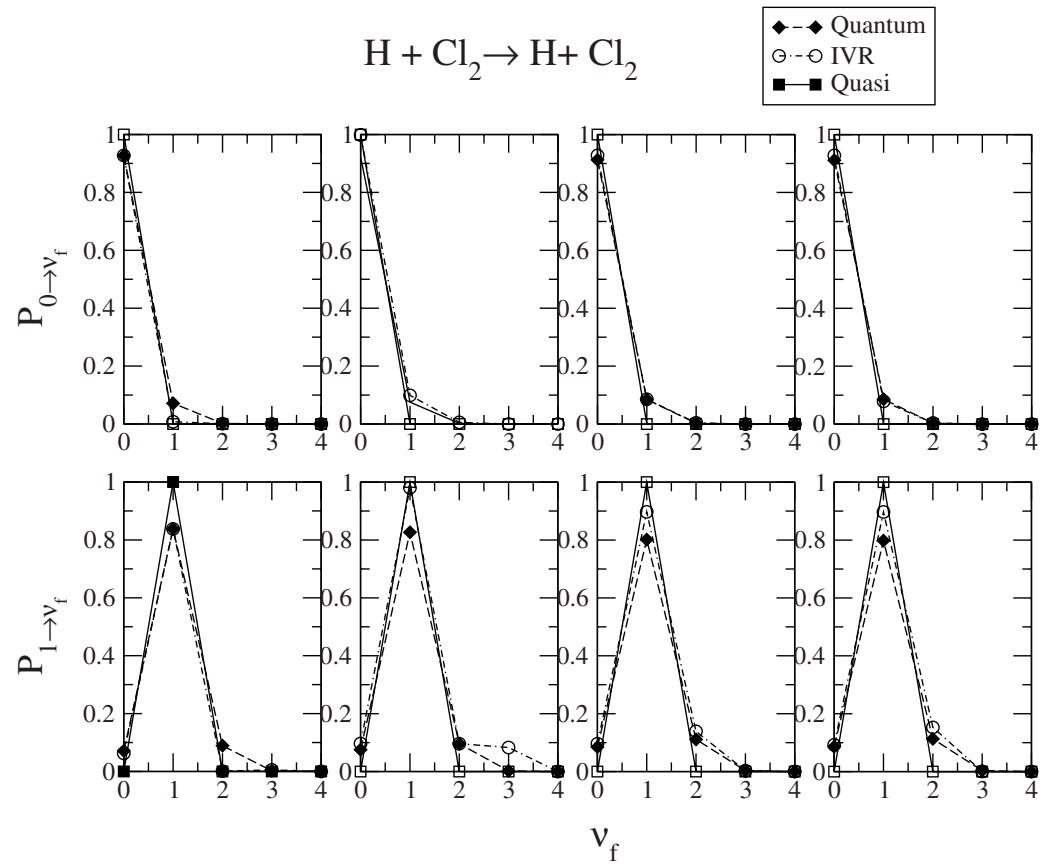

Fig. 1. PVDs of the semiclassical IVR inon reactive probabilities (empty squares connected by dashed-dotted lines $P_{0 \rightarrow \nu^{\prime}}\left(E_{t r}\right)$ (upper panel) and $P_{1 \rightarrow \nu^{\prime}}\left(E_{t r}\right)$ (lower panel) calculated at $\nu_{i}=0$ (upper panel) and $\nu_{i}=1$ (lower panel) at $E_{t r}=0.02,0.03$, 0.052 and $1.26 \mathrm{kcal} \mathrm{mol}^{-1}$ (from the left hand side to the right hand side)for the $\mathrm{H}+$ $\mathrm{Cl}_{2}\left(\nu_{i}\right) \rightarrow \mathrm{HCl}\left(\nu_{f}\right)+\mathrm{Cl}$. For comparison also quantum (diamonds connected by dashed lines) and quasiclassical (solid squares connected by solid lines) results are shown. 


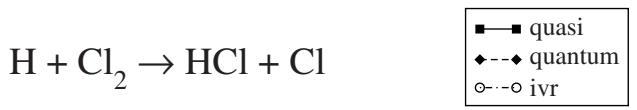
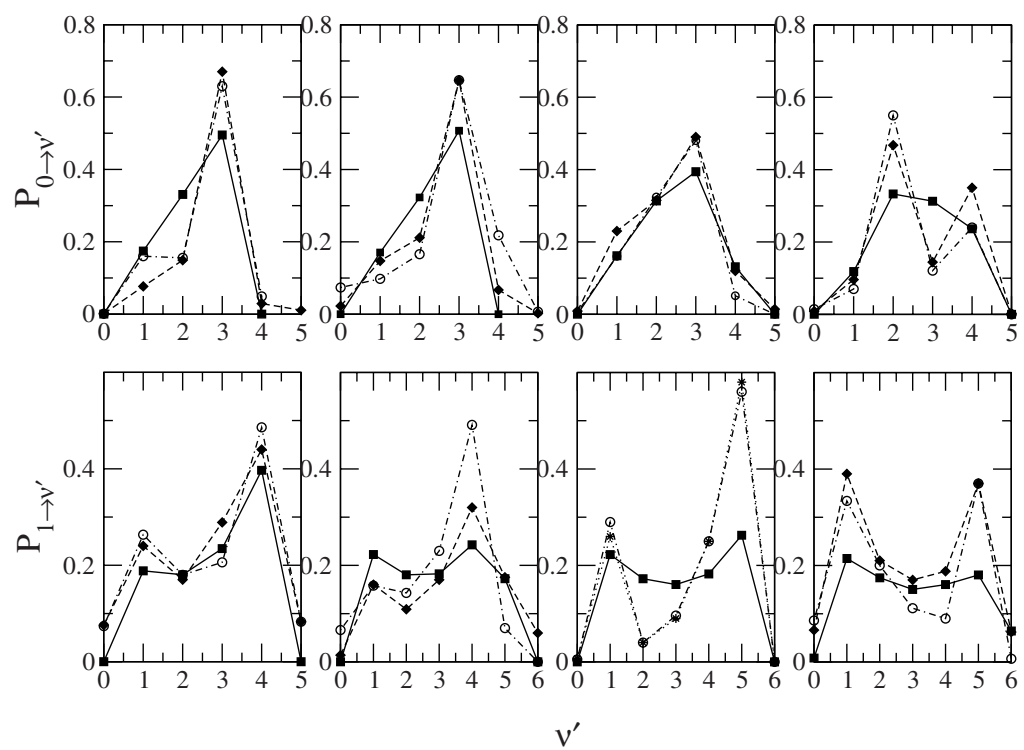

Fig. 2. PVDs of the semiclassical IVR reactive probabilities (empty squares connected by dashed-dotted lines $P_{0 \rightarrow \nu^{\prime}}\left(E_{t r}\right)$ (upper panel) and $P_{1 \rightarrow \nu^{\prime}}\left(E_{t r}\right)$ (lower panel) calculated at $\nu_{i}=0$ (upper panel) and $\nu_{i}=1$ (lower panel) at $E_{t r}=3,4,5$ and $6 \mathrm{kcal} \mathrm{mol}^{-1}$ (from the left hand side to the right hand side) for the $\mathrm{H}+\mathrm{Cl}_{2}\left(\nu_{i}\right) \rightarrow \mathrm{HCl}\left(\nu_{f}\right)+\mathrm{Cl}$. For comparison also quantum (diamonds connected by dashed lines) and quasiclassical (solid squares connected by solid lines) results are shown.

\section{References}

1. Miller, W. H., J. Phys. Chem. A 105, 2942 (2001).

2. Miller, W. H., J. Chem. Phys. 53, 3678 (1970).

3. Miller, W. H. and T. F. George, J. Chem. Phys. 56, 5668 (1972).

4. Goldstein, H., Classical Mechanics, Addison-Wesley, Massachusetts, 1970.

5. Skinner, D. E and Miller, W. H., Chem. Phys. Lett. 300, 20 (1999).

6. Lagañ̀, A., Gazzetta Chimica Italiana 111, 459 (1981).

7. Abramowitz, M. and Stegun, I. A., Handbook of Mathematical Functions, Dover, 1970.

8. Faginas Lago, N., PhD Thesis, University of Perugia, Perugia (2002) 